\title{
Arginine behaviour after arginine or citrulline administration in older subjects
}

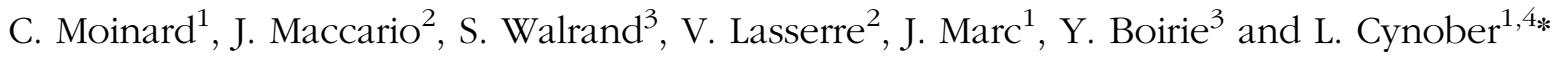 \\ ${ }^{1}$ Laboratoire de Biologie de la Nutrition EA 4466, Faculté des Sciences Pharmaceutiques et Biologiques de l'Université Paris \\ Descartes, 4 avenue de l'Observatoire, 75270 Paris Cedex 06, France \\ ${ }^{2}$ Laboratoire de Biomathématiques, Faculté de Pharmacie, Université Paris Descartes, Paris, 75006, France \\ ${ }^{3}$ UMR1019 Métabolisme protéino-énergétique, INRA, Université Clermont 1, Clermont-Ferrand, 63001, France \\ ${ }^{4}$ Service interhospitalier de Biochimie, Hopitaux Cochin \& Hôtel-Dieu, Hopitaux Universitaires Paris Centre (AP-HP), Paris, \\ 75014, France
}

(Submitted 30 April 2015 - Final revision received 19 October 2015 - Accepted 20 October 2015-First published online 1 December 2015)

\section{Abstract}

Arginine (ARG) and its precursor citrulline (CIT) are popular dietary supplements, especially for the elderly. However, age-related reductions in lean body mass and alterations in organ functions could change their bioavailability. Pharmacokinetics and tolerance to amino acid (AA) loads are poorly documented in elderly subjects. The objective here was to characterise the plasma kinetics of CIT and ARG in a single-dosing study design. Eight fasting elderly men underwent two separate isomolar oral loading tests (10 g of CIT or 9.94 g of ARG). Blood was withdrawn over an 8-h period to measure plasma AA concentrations. Only CIT, ornithine and ARG plasma concentrations were changed. Volume of distribution was not dependent on AA administered. Conversely, parameters related to ARG kinetics were strongly dependent on AA administered: after ARG load, elimination was higher (ARG > CIT; $P=0 \cdot 041$ ) and admission period + time at peak concentration was lower (ARG < CIT; $P=0.033$ ), and the combination of both phenomena results in a marked increase in ARG availability when CIT was administered (ARG < CIT; $P=0.033$ ) compared with ARG administration itself. In conclusion, a single CIT administration in the elderly is safe and well tolerated, and CIT proves to be a better in vivo ARG precursor than ARG itself in healthy elderly subjects.

\section{Key words: Pharmacokinetics: Amino acids: Ornithine: Food supplements}

Arginine (ARG) is known to be a versatile amino acid (AA), and its metabolic properties have been extensively studied ${ }^{(1)}$. ARG is largely used at pharmacological dosages in pathological states (e.g. injured patients) and as a food supplement in healthy subjects (athletes, elderly). ARG is proposed to increase growth hormone secretion and to sustain nitric oxide production. Pharmacokinetics studies have thus been led to characterise ARG behaviour in plasma after oral administration and to clarify its mechanisms of action ${ }^{(2)}$. It has also been proposed that citrulline (CIT) administration (a direct ARG precursor) could be a good alternative to further increase plasma ARG concentrations $^{(3-5)}$, as several experimental and clinical studies have found that CIT efficiently increases plasma ARG concentrations ${ }^{(3,6,7)}$. The metabolic pathways of ARG and CIT are closely inter-related, and metabolic channelling along with the respective complex inter-organ metabolisms ${ }^{(1,5,8)}$ may profoundly modify ARG metabolism and bioavailability according to original source (i.e. ARG or CIT). However, the available pharmacokinetic data refer solely to healthy young adults. Elderly people generally display a reduced lean body mass and alteration of organ functions (i.e. kidney and intestine, which have a key role in ARG and CIT metabolism), which means that what is true in young adults may not necessarily be true in the elderly. Even if ARG and CIT pharmacokinetics are well described in adult populations, there are still no data in elderly populations. We hypothesise that CIT is more suitable than ARG itself to generate ARG at the systemic level in the elderly. Accordingly, the aim of this study was to determine tolerance and pharmacokinetic characteristics after ARG or CIT administration in healthy older subjects, with special focus on systemic bioavailability of ARG according to original-source AA administered (i.e. ARG or CIT).

\section{Methods}

The study was approved by the Auvergne Region Institutional Review Board (IRB) and performed to the ethical standards of the Declaration of Helsinki. The protocol was not registered at http://clinicaltrials.gov, as it was accepted by the IRB before http://clinicaltrials.gov had gone online. All volunteers gave written informed consent after a full explanation of the study. One of the institutions involved in the study has French Ministry of Health authorisation to perform experiments on healthy volunteers.

\footnotetext{
Abbreviations: AA, amino acid; ARG, arginine; CIT, citrulline.
}

* Corresponding author: L. Cynober, fax +33 4537399 52, email luc.cynober@parisdescartes.fr 


\section{Subjects}

Volunteers were pre-selected from the General Clinical Research Center database. The study was performed on eight healthy elderly male volunteers (age $70 \cdot 2$ (sEm 1.2 ) years; BMI $\left.24.8(\operatorname{sem} 0.5) \mathrm{kg} / \mathrm{m}^{2}\right)$. The number of volunteers was calculated according to the results obtained with previous study ${ }^{(3)}$, particularly the variability of primary outcome. We calculated, with a risk of first species $\alpha=5 \%$ and second species $\beta=20 \%$, the number of subjects necessary to obtain a significant change in the AUC of plasma levels of AA. Under these conditions, a staff of eight subjects was considered as sufficient. All volunteers were given a medical check-up to ensure that they had no acute or chronic diseases or signs of infection and inflammation; none of the subjects were taking any medication liable to affect AA metabolism. Subjects were screened by physical examination, blood tests, urinary analysis and electrocardiogram according to EURAGE Senieur protocol admission criteria $^{(9)}$.

All volunteers received a normoproteic diet during the week before beginning the study and throughout the study.

\section{Protocol}

All volunteers received two isomolar oral loads consisting of CIT (10 g) and ARG (9.94 g) administered in random order, with each load separated by a washout period of $15 \mathrm{~d}$. CIT and ARG were dissolved into $150 \mathrm{ml}$ of water, and the solution was drunk rapidly. The glass was then rinsed in $50 \mathrm{ml}$ of water, again rapidly drunk by the volunteers. The doses are similar to those used in previous pharmacokinetics and therapeutic studies on these two AA or other related AA (e.g. ornithine $(\mathrm{ORN}))^{(2)}$. Blood samples were withdrawn just before administration (considered as time $0 \mathrm{~h}$ ) and at 0.25, 0.5, 0.75, 1, 1.5, 2, 3, 5 and $8 \mathrm{~h}$ after the loads. Urine samples (for the determination of $\mathrm{N}$ excretion) were collected in vessels containing antiseptic during the $16 \mathrm{~h}$ before load and then at $0-8 \mathrm{~h}$ after load.

\section{Measurements}

Plasma amino acid concentrations. Blood samples were rapidly centrifuged and deproteinised with a $30 \%$ (w/v) sulfosalicylic acid solution. The supernatant fractions were stored at $-80^{\circ} \mathrm{C}$ until analysis. AA were separated and quantified by ion exchange chromatography using an AA autoanalyzer (Aminotac JLC-500/V; Jeol Ltd) with ninhydrin derivatisation, as described previously ${ }^{(3)}$. Our participation in the European Quality Control Scheme (ERNDIM) is evidence of the accuracy of our AA determinations.

Nitrogen excretion. $\mathrm{N}$ was quantified by chemiluminescence on an Antek 9000 analyzer (Antek) ${ }^{(10)}$.

Other parameters. Biochemical markers (Na, K, Cl, urea, creatinine, alkaline phosphatase, alanine aminotransferase, aspartate aminotransferase, $\gamma$-glutamyltranspeptidase, total cholesterol, HDL-cholesterol, LDL-cholesterol, TAG, glucose, albumin, C-reactive protein) and haematological markers (leucocytes, polymorphonuclear cells, lymphocytes, monocytes, erythrocytes, prothrombin) were determined before and after the study period, as previously described ${ }^{(3)}$.

Pharmacokinetics and statistical analysis of plasma arginine after arginine or citrulline loads

Pharmacokinetic models and parameters. After various attempts, a model with a zero-order mechanism for the admission phase (i.e. appearance in systemic blood) and a firstorder elimination mechanism was chosen as giving the best fits to individual curves for ARG plasma levels. Each of these mechanisms may be understood as approximations of Michaelis-Menten monoenzymatic kinetics, the first being saturated and the second being far from saturation.

Plasma concentration is then described by the following relation:

$$
C(t)\left\{\begin{array}{cc}
B\left(1-\exp \left(-k_{E} t\right)\right)+b_{0} & \text { if } t \leq T_{A} \\
B\left(\exp \left(k_{E} T_{A}\right)-1\right) \exp \left(-k_{E} t\right)+b_{0} & \text { if } t>T_{A}
\end{array}\right\} .
$$

where $B$ is a 'size' parameter dependent on the dose and volume of distribution, $k_{E}$ is the parameter governing kinetic elimination, related to elimination half life $t_{1 / 2}$ by the usual rate constant relation $k_{E}=0.693 / t_{1 / 2}, T_{A}$ is the duration of the zeroorder admission kinetic, related to the duration of systemic appearance (probably because of the duration of transit through the part of the bowel where admission took place), $b_{0}$ is a baseline parameter to account for the non-zero physiological level of ARG.

To enable comparative analysis, secondary parameters were derived:

- AUC: $\operatorname{AUC}(0, \infty)=B T_{A}$

- time to reach peak concentration : $t_{\max }=T_{A}$

where peak concentration $C_{\max }=B\left(1-\exp \left(-k_{E} T_{A}\right)\right)+b_{0}$.

Statistical analysis. The statistical analysis was led in two steps: first, least square fitting of the model to individual curves to obtain primary and secondary parameters with assessments of their estimation precisions; second, comparison of the parameters between the two sets of values corresponding to either CIT or ARG administrations, which was conducted as simple paired comparisons for each parameter.

All computations were performed with $\mathrm{R}$, with the functions optimO for the first part and classical tests (Student, Wilcoxon or ANOVA) for the second part ${ }^{(11)}$.

\section{Results}

Seven of the eight subjects completed the two trials. There was one dropout (for personal reasons and not because of the study) who was not replaced. Subject characteristics are reported in Table 1.

\section{Tolerance}

None of the volunteers suffered nausea, diarrhoea or any other side effects, regardless of the trial. 
Table 1. Characteristics of the subjects (Mean values with their standard errors)

\begin{tabular}{lcl}
\hline & Mean & SEM \\
\hline Age (years) & 70.2 & 1.2 \\
Weight $(\mathrm{kg})$ & 71.3 & 2.7 \\
Size $(\mathrm{m})$ & 1.69 & 0.02 \\
BMl $\left(\mathrm{kg} / \mathrm{m}^{2}\right)$ & 24.8 & 0.5 \\
C-reactive protein $(\mathrm{mg} / \mathrm{l})$ & 1.87 & 0.57 \\
Albumin $(\mathrm{g} / \mathrm{l})$ & 39.1 & 0.7 \\
Glucose $(\mathrm{mmol} / \mathrm{l})$ & 4.76 & 0.21 \\
Cholesterol $(\mathrm{mmol} / \mathrm{l})$ & 5.43 & 0.24 \\
TAG $(\mathrm{mmol} / \mathrm{l})$ & 1.12 & 0.18 \\
\hline
\end{tabular}

Safety

A clinical and biological check-up was performed in order to evaluate the potential adverse effect of ARG or CIT load. None of the biomarkers measured were affected by ARG or CIT supplementation, and no clinical symptoms manifested during the study (data not shown).

\section{Urinary nitrogen excretion}

$\mathrm{N}$ excretion was similar before and after ARG load (0.43 (SEM $0.02)$ and $0.50(\operatorname{sem~} 0 \cdot 14) \mathrm{g} \mathrm{N} / \mathrm{h}$, respectively) or CIT load (0.48 (sem 0.08$)$ and $0.48(\operatorname{sem~} 0 \cdot 12) \mathrm{g} \mathrm{N} / \mathrm{h}$, respectively).

\section{Pharmacokinetic parameters of plasma arginine after oral} arginine or citrulline administration

The model gives a satisfactory fit of plasma ARG levels for both CIT load and ARG load (Fig. 1 gives the kinetics for subject no. 1 as a representative example). A bi-compartmental model was unable to reproduce the sharpness of the max of the curves and constantly gave poorer fits than the model used here (data not shown).

\section{Estimation of pharmacokinetic parameters}

For each pharmacokinetic parameter, the fit of the individual curves gave the parameter values below, reported with $\mathrm{CV}$ ( $\mathrm{CV}=$ asymptotic sD/estimated value).

\section{$B$ (Table 2)}

For subject no. 4 , the value of the $B$ parameter for the ARG group $(2 \cdot 1520)$ is probably an outlier. Accordingly, parametric (paired Student) and non-parametric (paired Wilcoxon) test comparisons were performed. Only the Student's test without subject no. 4 gave a $P$ value $<5 \%(0.0489)$, but it was so close that we concluded that the difference was not significant.

\section{$k_{E}$ (Table 3)}

There was a slightly significant difference between the two paired sets of $k_{E}$ parameters (paired Student's test; $P=4.1 \%$ ), which confirmed that the rate of ARG elimination differed between the two groups.

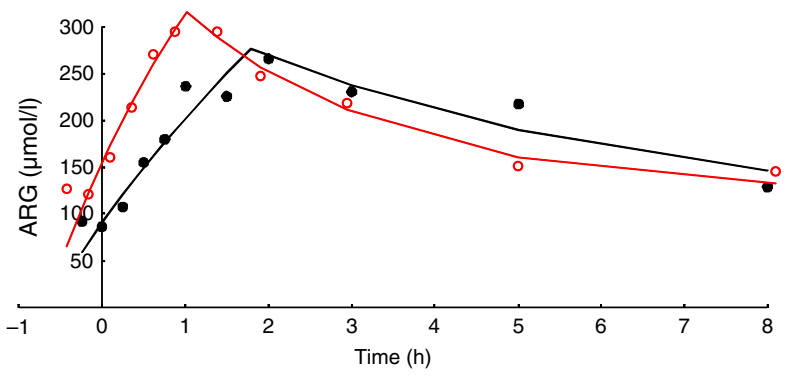

Fig. 1. Time course of plasma arginine (ARG) after oral citrulline load (10 g; O) $v$. after oral ARG load (9.94 g; ) (kinetics of subject no. 1 shown here as a representative example). Means of experimental measures $(\mathcal{O})$ and estimated values (-).

Table 2. Results of the fits of the pharmacokinetic model for parameter $B$ ('size' parameter dependent on dose and volume of distribution) (Estimations and coefficients of variation)

\begin{tabular}{lccccc}
\hline & \multicolumn{2}{c}{ ARG group } & & \multicolumn{2}{c}{ CIT group } \\
\cline { 2 - 3 } \cline { 5 - 6 } Subjects & $B$ & CV $(B)(\%)$ & & $B$ & CV $(B)(\%)$ \\
\hline S1 & 0.4961 & 16.6 & & 0.6354 & 32.9 \\
S2 & 0.6317 & 35.2 & & 0.5241 & 49.9 \\
S3 & 0.4868 & 30.8 & & 0.7028 & 30.2 \\
S4 & 2.1520 & 55.4 & & 0.5151 & 22.6 \\
S5 & 0.3589 & 30.9 & & 0.7590 & 15.1 \\
S6 & 0.4081 & 56.7 & & 1.1210 & 32.0 \\
S7 & 0.5246 & 17.5 & & 1.2140 & 19.4 \\
\hline
\end{tabular}

ARG, arginine; CIT, citrulline.

Table 3. Results of the fits of the pharmacokinetic model for parameter $k_{E}$ (parameter governing kinetic elimination)

(Estimations and coefficients of variation)

\begin{tabular}{lccccc}
\hline & \multicolumn{2}{c}{ ARG group } & & \multicolumn{2}{c}{ CIT group } \\
\cline { 2 - 3 } \cline { 5 - 6 } Subjects & $k_{E}$ & CV $\left(k_{E}\right)(\%)$ & & $k_{E}$ & CV $\left(k_{E}\right)(\%)$ \\
\hline S1 & 0.41700 & 15.9 & & 0.1935 & 29.6 \\
S2 & 0.32200 & 27.2 & & 0.1474 & 50.1 \\
S3 & 0.21890 & 28.9 & & 0.1541 & 29.6 \\
S4 & 0.07412 & 50.0 & & 0.1989 & 26.9 \\
S5 & 0.36240 & 29.8 & & 0.1310 & 13.7 \\
S6 & 0.29440 & 50.9 & & 0.1091 & 29.5 \\
S7 & 0.60580 & 17.4 & & 0.1490 & 18.1 \\
\hline
\end{tabular}

ARG, arginine; CIT, citrulline.

$T_{\text {A }}($ Table 4)

There was a significant difference between the two paired sets of $T_{A}$ parameters (paired Student's test; $P=3 \cdot 3 \%$ ), with length of the admission period and time at peak concentration being larger in the CIT group.

\section{$b_{0}$ (Table 5)}

At baseline $\left(b_{0}\right)$, plasma levels of ARG, as expected, were not significantly different between the two groups (paired Student's test; $P=96 \%)$. 
Table 4. Results of the fits of the pharmacokinetic model for parameter $T_{A}$ (duration of the zero-order admission kinetic, related to the duration of systemic appearance)

(Estimations and coefficients of variation)

\begin{tabular}{lccccc}
\hline & \multicolumn{2}{c}{ ARG group } & & \multicolumn{2}{c}{ CIT group } \\
\cline { 2 - 3 } \cline { 5 - 6 } Subjects & $T_{A}$ & CV $\left(T_{A}\right)(\%)$ & & $T_{A}$ & CV $\left(T_{A}\right)(\%)$ \\
\hline S1 & 1.1360 & 7.14 & & 1.790 & 13.40 \\
S2 & 0.7500 & 15.80 & & 2.296 & 20.70 \\
S3 & 1.5800 & 13.00 & & 2.532 & 12.40 \\
S4 & 0.8362 & 16.60 & & 3.680 & 11.80 \\
S5 & 1.8040 & 12.90 & & 1.850 & 5.70 \\
S6 & 1.1340 & 23.10 & & 1.838 & 11.40 \\
S7 & 1.3770 & 7.19 & & 1.582 & 7.49 \\
\hline
\end{tabular}

ARG, arginine; CIT, citrulline.

Table 5. Results of the fits of the pharmacokinetic model for parameter $b_{0}$ (baseline parameter to account for the non-zero physiological level of arginine (ARG))

(Estimations and coefficients of variation)

\begin{tabular}{lccccc}
\hline & \multicolumn{2}{c}{ ARG group } & & \multicolumn{2}{c}{ CIT group } \\
\cline { 2 - 3 } \cline { 5 - 6 } Subjects & $b_{0}$ & CV $\left(b_{0}\right)(\%)$ & & $b_{0}$ & CV $\left(b_{0}\right)(\%)$ \\
\hline S1 & 0.08251 & 7.04 & & 0.09084 & 13.10 \\
S2 & 0.07539 & 11.70 & & 0.10700 & 11.20 \\
S3 & 0.09305 & 9.24 & & 0.07296 & 15.40 \\
S4 & 0.09847 & 12.60 & & 0.09642 & 8.85 \\
S5 & 0.10680 & 9.92 & & 0.09683 & 4.51 \\
S6 & 0.09403 & 13.80 & & 0.08766 & 12.30 \\
S7 & 0.10930 & 9.05 & & 0.11010 & 8.28 \\
\hline
\end{tabular}

CIT, citrulline.

$\mathrm{C}_{\max }($ Table 6)

There was no significant between-group difference in $C_{\max }$ (paired Student's test; $P=14 \%$ ).

\section{AUC (Table 7)}

The AUC of plasma ARG (Table 7) was significantly larger (paired one-sided Student's test; $P=0.6 \%$ ) after CIT administration than after ARG administration.

\section{Summary of the results (Table 8)}

Volume of distribution (parameter $B$ ) and baseline ARG level (parameter $b_{0}$ ) were not dependent on AA administered (i.e. ARG or CIT load). Conversely, the parameters related to ARG kinetics were strongly dependent on AA administered: $k_{E}$ was larger and $T_{A}$ was shorter when ARG was administered. The higher elimination $\left(k_{E} ; P=4.1 \%\right)$ of ARG and lower admission period and time at peak concentration $\left(T_{A} ; P=3.3 \%\right)$ combine to give markedly lower ARG bioavailability when ARG itself is administered than when CIT is administered.

\section{Discussion}

AA are very popular with many populations because of their (real or perceived) properties, and they are widely consumed as dietary supplements. ARG certainly counts among the most
Table 6. Results of the fits of the pharmacokinetic model for parameter $C_{\max }$ (peak concentration)

(Estimations and coefficients of variation)

\begin{tabular}{llcccc}
\hline & \multicolumn{2}{c}{ ARG group } & & \multicolumn{2}{c}{ CIT group } \\
\cline { 2 - 3 } \cline { 5 - 6 } Subjects & $C_{\max }$ & CV $\left(C_{\max }\right)$ & & $C_{\max }$ & CV $\left(C_{\max }\right)$ \\
\hline S1 & 0.2697 & 2.96 & & 0.2768 & 5.29 \\
S2 & 0.2109 & 4.47 & & 0.2575 & 7.93 \\
S3 & 0.2354 & 5.09 & & 0.3000 & 5.61 \\
S4 & 0.2278 & 5.24 & & 0.3638 & 4.43 \\
S5 & 0.2790 & 4.56 & & 0.2602 & 2.06 \\
S6 & 0.2098 & 7.89 & & 0.2914 & 4.62 \\
S7 & 0.4062 & 3.02 & & 0.3650 & 3.48 \\
\hline
\end{tabular}

ARG, arginine; CIT, citrulline.

Table 7. Results of the fits of the pharmacokinetic model for the parameter AUC

(Estimations and coefficients of variation)

\begin{tabular}{lccccc}
\hline & \multicolumn{2}{c}{ ARG group } & & \multicolumn{2}{c}{ CIT group } \\
\cline { 2 - 3 } \cline { 5 - 6 } Subjects & $A U C(0, \infty)$ & $C V(A \cup C(0, \infty))$ & & $A U C(0, \infty)$ & $C V(A \cup C(0, \infty))$ \\
\hline S1 & 0.5635 & 11.8 & & 1.1371 & 24.4 \\
S2 & 0.4737 & 24.5 & & 1.2035 & 35.6 \\
S3 & 0.7692 & 22.8 & & 1.7793 & 21.6 \\
S4 & 1.7994 & 46.4 & & 1.8959 & 13.6 \\
S5 & 0.6474 & 22.6 & & 1.4040 & 11.5 \\
S6 & 0.4627 & 41.9 & & 2.0600 & 24.8 \\
S7 & 0.7224 & 12.4 & & 1.9205 & 14.7 \\
\hline
\end{tabular}

ARG, arginine; CIT, citrulline.

popular and most widely consumed, but CIT, an efficient ARG precursor, is also largely used. Several studies have already evaluated the pharmacokinetics of these two AA in healthy adults $^{(2-4,12)}$ and demonstrated the ability of CIT to generate ARG. However, to the best of our knowledge, there has been no assessment in older populations, despite the fact that ageing is associated with decline in organ functions, particularly the kidneys and intestine, which have a pivotal role in CIT and ARG metabolism: CIT is synthesised from ARG in the small bowel and ARG from CIT in the kidneys ${ }^{(5)}$, which means any alteration in the functionality of these organs may affect ARG and CIT metabolism and tolerance to ARG and CIT supplementation ${ }^{(8)}$. It is well known that ORN $\alpha$-ketoglutarate, an ARG precursor, is well tolerated in young adults up to a $10-\mathrm{g} \mathrm{load}^{(13)}$, whereas this same dose often triggers diarrhoea in elderly subjects (S Allison, personal communication).

The results obtained here clearly indicate that CIT and ARG at this selected dosage are well tolerated in the elderly, as neither induced gastrointestinal disorders, which was not evident for $\mathrm{ARG}^{(14)}$. With regard to the safety of CIT in the elderly, biological exploration did not show any side effect of acute administration of this AA. This result is in line with previous studies in young adults and confirms the safety of $\mathrm{CIT}^{(3,5)}$.

The two main sites of endogenous ARG synthesis from CIT are the liver, where ARG is synthesised and then immediately hydrolysed within the urea cycle into $\mathrm{ORN}^{(15)}$, and the renal cortex, where most of the synthesised ARG is released into the bloodstream $^{(3)}$. The present work clearly indicates in the elderly 
Table 8. Summary of the tests of pharmacokinetic parameters

\begin{tabular}{lcccl}
\hline & ARG group & CIT group & & \\
\hline$B$ & 0.7817 & 0.7226 & $\mathrm{NS}$ & \\
$k_{E}$ & 0.1547 & 0.3280 & $\mathrm{~S}$ & $\mathrm{ARG}>\mathrm{CIT}$ \\
$T_{A}=t_{\max }$ & 2.2239 & 1.2309 & $\mathrm{~S}$ & $\mathrm{ARG}<\mathrm{CIT}$ \\
$C_{\max }$ & 0.3021 & 0.2627 & $\mathrm{NS}$ & \\
$b_{0}$ & 0.0945 & 0.0942 & $\mathrm{NS}$ & \\
$A \cup C(0, \infty)$ & 1.6286 & 0.7769 & $\mathrm{~S}$ & $\mathrm{ARG}<\mathrm{CIT}$ \\
\hline
\end{tabular}

ARG, arginine; CIT, citrulline; S, significant.

that CIT is an ARG precursor at the whole-body level. Of note, the $C_{\max }$ of ARG after CIT load appears to be the same as previously observed in young adults (i.e. approximately $280 \mu \mathrm{mol} / \mathrm{l})^{(3)}$. Such an observation is in agreement with the experimental work performed by Mistry et al. ${ }^{(8)}$, which clearly demonstrated that the expression levels of the genes involved in renal ARG synthesis and intestinal CIT synthesis are not affected by ageing.

In addition, the ARG generated gets very rapidly metabolised into ORN, confirming that in the elderly, as in adults, arginase activity is not a limiting step in urea synthesis, whereas experimental data using isolated perfused rat liver indicated a drop in urea production in the liver of old animals compared with young ones ${ }^{(16)}$. Note, however, that these ex vivo studies fail to integrate inter-organ regulation.

However, the data obtained raise the question of why ARG synthesised from exogenous CIT does not have the same behaviour as ARG from exogenous ARG. Our data suggest a better bioavailability of CIT, which is supported by the fact that CIT is absorbed by the intestinal brush border by a broad array of transporters including the $\mathrm{B} 0,+, \mathrm{b} 0,+$ and $\mathrm{L}$ systems ${ }^{(17)}$. However, this is not the only factor involved in ARG bioavailability after CIT load. In a recent experimental study ${ }^{(18)}$, we observed that CIT bioavailability decreased considerably in the course of endotoxaemia, whereas ARG generation remain unmodified, confirming that CIT intestinal absorption is not the only mechanism involved. Clearly, such a result confirms our hypothesis that CIT is a better ARG precursor than ARG itself, and the pharmacokinetic study reported here in elderly subject confirms results from previous experimental data ${ }^{(5)}$ and clinical data $^{(3-5,19)}$ obtained in young adults.

In an attempt to define a suitable pharmacokinetic model of plasma ARG concentrations after ARG or CIT load, we can consider that the appearance and elimination rates of AA in plasma are both controlled by cellular carriers or enzymes with a specific constant - that is intestinal transport and arginase activity in tissues other than hepatocytes where arginase activity is never limiting ${ }^{(16)}$. We ran an attempt with a double MichaelisMenten model, but the relatively small number of observation time points did not enable satisfactory fits. We consequently worked on approximations of this model. Usually, when an enzyme is confronted with decreasing concentrations of substrate, as the concentration becomes smaller than the affinity constant $K m$ of the enzyme, the process may be fairly described by a first-order kinetics. This kind of approximation also appears to be valid for the elimination process. Admission instead is better described by a zero-order process, which means that the substrate concentrations are higher than the $\mathrm{Km}$ of the enzyme (observed values are approximately $15-30 \mathrm{~mm}$, whereas the $\mathrm{Km}$ of arginases is approximately $0.1 \mathrm{~mm}$ for Arginase 1 and $5 \mathrm{~mm}$ for Arginase $2^{(20,21)}$ ). In usual practice, this kind of zero-order process is only transient and the mechanism evolves to a first-order kinetic when concentration decreases. The explanation could be that a bolus AA solution is at a much higher concentration than physiological levels, and one can hypothesise that the admission process takes place on a limited length of the intestinal tract that does not allow the plasma concentration to fall below the $\mathrm{Km}$, with the result that admission process thus stays close to its zero-order approximation. Such a hypothesis obviously needs to be confirmed by tracer studies, allowing us to explore more precisely the ARG metabolism (and in particular metabolic flux controlled by arginases or nitric oxide synthase).

In conclusion, the present pharmacokinetic study clearly shows in the elderly that CIT is a potent ARG precursor at the whole-body level, thus confirming that ARG availability does not decline with advanced age. Moreover, for the first time, CIT and ARG pharmacokinetics are compared in the same study, and we confirm that plasma ARG behaviour differs markedly according to the molecule (CIT or ARG) administered. Furthermore, a 10-g dose of CIT is well tolerated in the elderly, and it demonstrates metabolic advantages over ARG in terms of bioavailability, suggesting that CIT supplementation is suitable in the elderly even for ARG supplementation (i.e. for CVD) ${ }^{(22)}$. Of note, the therapeutic advantage remains to be determined.

\section{Acknowledgements}

This work was funded by the French Ministry of Research and Technology (EA 4466).

L. C. and C. M. designed the study, and C. M. wrote the manuscript. All the other authors contributed to the manuscript and to the discussion. S. W. and Y. B. performed the clinical study, J. M. collected the data and performed amino acid analysis and J. M. and V. L. performed statistical analysis and modelisation. All authors read and approved the final manuscript.

L. C. and C. M. are shareholders of Citrage Company.

\section{References}

1. Wu G, Bazer FW, Davis TA, et al. (2009) Arginine metabolism and nutrition in growth, health and disease. Amino Acids 37, 153-168.

2. Cynober L (2007) Pharmacokinetics of arginine and related amino acids. $J$ Nutr 137, 1646S-1649S.

3. Moinard C, Nicolis I, Neveux N, et al. (2008) Dose-ranging effects of citrulline administration on plasma amino acids and hormonal patterns in healthy subjects: the Citrudose pharmacokinetic study. Br J Nutr 99, 855-862.

4. Schwedhelm E, Maas R, Freese R, et al. (2007) Pharmacokinetic and pharmacodynamic properties of oral L-citrulline and L-arginine: impact on nitric oxide metabolism. $\mathrm{Br} J$ Clin Pharmacol 65, 51-59.

5. Bahri S, Zerrouk N, Aussel C, et al. (2013) Citrulline: from metabolism to therapeutic use. Nutrition 29, 479-484. 
6. Osowska S, Moinard C, Neveux N, et al. (2004) Citrulline increases arginine pools and restores nitrogen balance after massive intestinal resection. Gut 53, 1781-1786.

7. Lassala A, Bazer FW, Cudd TA, et al. (2009) Intravenous administration of L-citrulline to pregnant ewes is more effective than L-arginine for increasing arginine availability in the fetus. J Nutr 139, 660-665.

8. Mistry SK, Greenfeld Z, Morris SM, et al. (2002) The 'intestinalrenal' arginine biosynthetic axis in the aging rat. Mech Ageing Dev 123, 1159-1165.

9. Ligthart GJ, Corberand JX, Fournier C, et al. (1984) Admission criteria for immunogerontological studies in man: the SENIEUR protocol. Mech Ageing Dev 28, 47-55.

10. Grimble G (1991) Measurement of elemental nitrogen by chemiluminescence. JPEN J Parenter Enteral Nutr 15, 696.

11. R Development Core Team (2005) A Language and Environment for Statistical Computing. Vienna: R Foundation for Statistical Computing.

12. Tangphao O, Grossmann M, Chalon S, et al. (1999) Pharmacokinetics of intravenous and oral L-arginine in normal volunteers. Br J Clin Pharmacol 47, 261-266.

13. Cynober L, Vaubourdolle M, Dore A, et al. (1984) Kinetics and metabolic effects of orally administered ornithine alphaketoglutarate in healthy subjects fed with a standardized regimen. Am J Clin Nutr 39, 514-519.

14. Grimble GK (2007) Adverse GI effects of arginine and related amino acids. J Nutr 137, 1693S-1701S.
15. Meijer AJ, Lamers WH \& Chamuleau RA (1990) Nitrogen metabolism and ornithine cycle function. Physiol Rev $\mathbf{7 0}$, 701-748.

16. Jourdan M, Cynober L, Moinard C, et al. (2008) Splanchnic sequestration of amino acids in aged rats: in vivo and ex vivo experiments using a model of isolated perfused liver. $A m$ J Physiol Regul Integr Comp Physiol 294, R748-R755.

17. Bahri S, Zerrouk N, Curis E, et al. (2005) Characterization of L-citrulline transport in human intestinal Caco-2 cells. Clin Nutr 24, 630.

18. Elwafi F, Curis E, Zerrouk N, et al. (2012) Endotoxemia affects citrulline, arginine and glutamine bioavailability. Eur J Clin Invest 42, 282-289.

19. Jourdan M, Nair KS, Carter RE, et al. (2015) Citrulline stimulates muscle protein synthesis in the post-absorptive state in healthy people fed a low-protein diet - a pilot study. Clin Nutr 34, 449-456.

20. Di Costanzo L, Sabio G, Mora A, et al. (2005) Crystal structure of human arginase I at 1.29-A resolution and exploration of inhibition in the immune response. Proc Natl Acad Sci US A 102, 13058-13063.

21. Durante W, Johnson FK \& Johnson RA (2007) Arginase: a critical regulator of nitric oxide synthesis and vascular function. Clin Exp Pharmacol Physiol 34, 906-911.

22. Lorin J, Zeller M, Guilland J-C, et al. (2014) Arginine and nitric oxide synthase: regulatory mechanisms and cardiovascular aspects. Mol Nutr Food Res 58, 101-116. 